\title{
MONOCULTIVO DE EUCALIPTO E CONSÓRCIO COM SESBÂNIA: CRESCIMENTO INICIAL EM CAVAS DE EXTRAÇÃO DE ARGILA ${ }^{1}$
}

\begin{abstract}
Anderson Ribeiro Santiago², Deborah Guerra Barroso ${ }^{3}$, Andrea Vita Reis Mendonça ${ }^{4}$, Eliemar Campostrini $^{3}$, José Geraldo de Araújo Carneiro ${ }^{3}$ e Edênio Detmann ${ }^{5}$

RESUMO - O consórcio de eucalipto com sesbânia na reabilitação de cavas de extração de argila pode representar uma forma de uso com benefícios ecológicos e econômicos, tendo em vista a sub-utilização a que essas cavas estão sendo submetidas. Este trabalho teve por objetivo avaliar a sobrevivência, o crescimento inicial e características fisiológicas de Eucalyptus camaldulensis, E. tereticornis, E. robusta e E. pellita, em monocultivos e plantios consorciados com Sesbania virgata. Foram instalados dois experimentos (monocultivo e plantio consorciado), numa cava de extração de argila, segundo o delineamento em blocos casualizados com quatro repetições. Os plantios consorciados favoreceram a sobrevivência das espécies. Os eucaliptos no monocultivo apresentaram maior crescimento inicial em diâmetro do colo e em área de copa. As espécies de eucalipto responderam aos efeitos do consórcio e das podas ao longo do tempo, exceto E. tereticornis.
\end{abstract}

Palavras-chave: Eucalyptus spp., monocultivo e plantio consorciado.

\section{EUCALYPTUS MONOCROPPING AND INTERCROPPED WITH SESBANIA: INITIAL GROWTH IN CLAY MINING DIGGINGS}

\begin{abstract}
Intercropping of Eucalyptus and sesbania for the recovery of clay mining diggings can represent ecological and economic benefits. This work aimed to evaluate survival, initial growth and physiological characteristics of Eucalyptus camaldulensis, E. tereticornis, E. robusta and E. pellita, monocropped and intercropped with Sesbania virgata. Two experiments (monocropping and intercropped) were set up in a clay mining digging, arranged in a randomized block design with 4 replicates. Intercropping favored the survival of the species. Eucalyptus monocropping presented a greater initial growth in soil level diameter and canopy area. The eucalyptus species responded to the effects of intercropping and pruning, along time, except for E. tereticornis.
\end{abstract}

Keywords: Eucalyptus spp., monocropping and intercropping.

\section{INTRODUÇÃO}

O impacto ambiental causado pela atividade de extração de argila, no Município de Campos dos Goytacazes, RJ, tem sido relatado por alguns autores (COSTAJÚNIOR, 1997; COUTINHO, 2003; SANTIAGO et al., 2004; SCHIAVO et al., 2004).

Estima-se que as cerâmicas da região retiram da camada subsuperficial do solo um total de $5.700 \mathrm{~m}^{3}$ $\mathrm{dia}^{-1}$ de matéria-prima. No entanto, geram cerca de 6.000 empregos diretos e movimentam em torno de $\mathrm{R} \$ 168$ milhões por ano, destacando-se o município como o segundo maior produtor de tijolos do Brasil (RAMOS et al., 2003).

A atividade de extração de argila causa, assim, sérios danos ambientais, ao mesmo tempo que gera empregos e movimenta uma economia importante.

\footnotetext{
${ }^{1}$ Recebido em 18.09.2007 e aceito para publicação em 26.01.2009.

${ }^{2}$ Instituto Natureza do Tocantins, Araguaína-TO. E-mail: <andersantiago@ibest.com.br>.

${ }^{3}$ Universidade Estadual do Norte Fluminense (UENF), Campos dos Goytacazes-RJ.E-mail: <deborah@ uenf.br>; <carneiro@ uenf.br>.

${ }^{4}$ Departamento de Engenharia Florestal da Universidade Federal do Recôncavo da Bahia (UFRB) - Cruz das Almas-BA.

E-mail: <avrmendonca@hotmail.com>.

${ }_{5}^{5}$ Departamento de Zootecnia da Universidade Federal de Viçosa (UFV). E-mail: <detmann @ ufv.br>.
} 
Segundo a Constituição Federal (Artigo 225, Parágrafo $2^{\circ}$ ), o explorador é obrigado a recuperar o ambiente degradado. O plantio de espécies florestais de uso múltiplo, que auxiliem a melhorias química e física das cavas, resultante de um ciclo mais longo de produção, pode representar uma possibilidade vantajosa de utilização dessas áreas, permitindo conciliar retorno econômico e sustentabilidade ao empreendimento.

É necessário, entretanto, avaliar o comportamento das espécies e sua adaptação às condições das cavas, identificando material com potencial produtivo.

Várias técnicas de plantio, como consórcio entre espécies, cultivo em aléias, conduzidas via podas, e sistemas agroflorestais, têm sido testadas em ambientes com algum estágio de degradação (AMADOR e VIANA, 1998; ARATO et al., 2003; MAFRA et al., 1998a; NÓBREGA et al., 2002). Os resultados indicaram aumento no teor de matéria orgânica no solo e, de maneira geral, na disponibilidade de nutrientes, além da diversificação da produção agrícola (CECCON et al., 1999).

Schiavo et al. (2004), avaliando o consórcio de Eucalyptus camaldulensis Dehn com Acacia mangium Willd, em cavas de extração de argila, constataram que o crescimento do eucalipto foi favorecido, entre outros fatores, pelo consórcio com a leguminosa arbórea. Rodrigues et al. (2003a), consorciando Eucalyptus grandis com Sesbania virgata (Cav.) Pers, em condições controladas, comprovaram, em todos os tratamentos, a transferência de $\mathrm{N}$ das plantas de sesbânia para o eucalipto.

Vezzani et al. (2001), avaliando o crescimento em altura e diâmetro de E. saligna (Smith), aos 45 meses, em plantios puros e consorciados com Acacia mearnsii (Wild.), no espaçamento de 4,0 x 1,5 m, não constataram diferenças entre os sistemas de plantio. Ao contrário, Parrota (1999), em monocultivos e plantios consorciados de Casuarina equestifolia, Eucalyptus robusta e Leucaena leucocephala, aos 8,5 anos de idade, verificou que a produtividade total de biomassa da parte aérea no consórcio do eucalipto com qualquer uma das outras espécies foi quase duas vezes maior que no seu monocultivo. Também, Baliero et al. (2004) observaram no consórcio de E. grandis com guachapele (Pseudosamanea guachapele), plantados em linhas

\footnotetext{
${ }^{1}$ Cava pertencente à Cerâmica Stilbe Ltda.
}

alternadas no espaçamento de $3 \times 1 \mathrm{~m}$, que as árvores de eucalipto apresentaram diâmetro $29 \%$ maiores no plantio consorciado, com relação ao monocultivo, aos 7 anos. No entanto, Jesus e Dias (1988), trabalhando com espaçamento menor (2,73 x 2,73 m), observaram que a produtividade em $\mathrm{m}^{3} \mathrm{ha}^{-1} \mathrm{ano}^{-1} \mathrm{de}$ E. urophylla Blake, aos sete anos, foi menor no consórcio com Leucaena leucocephala Lam.

O objetivo deste trabalho foi avaliar a sobrevivência e o crescimento inicial da parte aérea e do sistema radicular, bem como algumas características fisiológicas (massa específica foliar e estimativa de teor de clorofila, pelo medidor portátil de clorofila - MPC ou valor SPAD - Soil Plant Analysis Development) de Eucalyptus camaldulensis Dehn, E. tereticornis $\mathrm{Sm}$, E. robusta $\mathrm{Sm}$ e E. pellita F. Muell, em plantios puros e consorciados com Sesbania virgata (Cav.) Pers., numa cava resultante da extração de argila.

\section{MATERIAL E MÉTODOS}

Em uma cava de extração de $\operatorname{argila}^{1}$, localizada no distrito de Campo Limpo (21 ${ }^{\circ} 51^{\prime} \mathrm{S}, 41^{\circ} 14^{\prime} \mathrm{W}$, a altitude de $14 \mathrm{~m}$ ), Campos do Goytacazes, RJ, foram instalados dois experimentos.

A extração de argila é realizada, no município, em planície com material do período Quaternário, do Baixo Paraíba do Sul, local em que os solos apresentam grandes reservas de argila. Em trabalho de caracterização edáfica da área, Valicheski (2004) identificou o solo original como um Cambissolo Háplico Sódico gleico salino, formado a partir de sedimentos aluviais de uma sequiência variada de extratos do rio Paraíba do Sul, ocorrida no período quaternário (holoceno). O relevo apresentase plano.

Com 1 ha de extensão, a cava foi explorada no período de 1999 a 2002, a uma profundidade de extração variando de 2 a $2,5 \mathrm{~m}$. O lençol freático foi encontrado, no período seco, a 1,4 $\mathrm{m}$ de profundidade e, no período chuvoso, a $1 \mathrm{~m}$.

A temperatura média mensal do município variou, nos últimos 10 anos, entre $21,4^{\circ} \mathrm{C}$ e $27,7^{\circ} \mathrm{C}$. No período experimental, a temperatura média mensal variou de $18,3^{\circ} \mathrm{Ca} 28,2^{\circ} \mathrm{C}$, com precipitação de $767 \mathrm{~mm}$, distribuída conforme Figura 1. 


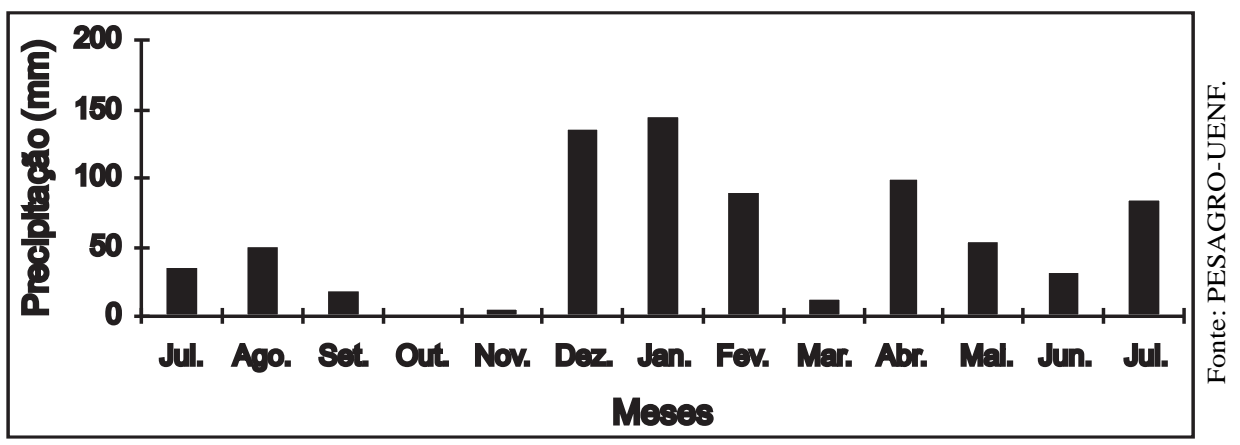

Figura 1 - Precipitação pluviométrica durante os meses de julho de 2003 a julho de 2004. Figure 1 -Rainfall during the months of July of 2003 to July of 2004

Monocultivos e plantios consorciados de eucalipto foram conduzidos em blocos casualizados, com quatro repetições, de 18 plantas. Os plantios foram realizados no mês de julho de 2003, sem nenhuma adubação de plantio ou de manutenção. No monocultivo, mudas de Eucalyptus camaldulensis Dehn, E. tereticornis $\mathrm{Sm}$, E. robusta $\mathrm{Sm}$ e E. pellita $\mathrm{F}$. Muell foram plantadas em covas, no espaçamento $3 \times 2 \mathrm{~m}$. No plantio consorciado, as mudas dessas espécies foram plantadas, no mesmo espaçamento, nas entrelinhas da leguminosa arbustiva Sesbania virgata (Cav.) Pers, que na época estava com altura média de 2,80 $\pm 0,12 \mathrm{~m}$, aos 12 meses de idade.

No consórcio, as plantas de sesbânia foram podadas aos 10, 16 e 22 meses, com duas finalidades: primeiro para maximizar a disponibilidade de nutrientes as plantas e, segundo, em razão de ter sido detectada uma tendência de estabilização no crescimento em altura e diâmetro do colo da leguminosa, aferida por Coutinho et al. (2005), aos 10 meses. O material resultante da poda foi distribuído, de forma homogênea, nas entrelinhas de plantio

Antes do plantio, para caracterização, foi coletada uma amostra do substrato por bloco, composta por quatro subamostras, em duas profundidades. Para análise granulométrica e caracterização química, as profundidades de amostragem foram: 0 a $20 \mathrm{~cm}$ e 20 a $40 \mathrm{~cm}$. Para porosidade, densidade da partícula e do substrato, as profundidades de amostragem foram: 0 a $10 \mathrm{~cm} \mathrm{e}$ 10 a $20 \mathrm{~cm}$. Na análise química, foram determinados $\mathrm{pH}$ (em água), acidez potencial, condutividade elétrica e carbono orgânico, segundo a metodologia descrita pela EMBRAPA (1997) e os teores de P, Ke Na extraíveis por Mehlich-1, Ca, Mg e Al trocáveis pelo $\mathrm{KCl} 1 \mathrm{~mol}$ $\mathrm{L}^{-1}$. Nas análises físicas, foram determinados, segundo métodos descritos em EMBRAPA (Op. cit.), densidade do substrato (anel volumétrico) e da partícula (balão volumétrico), porosidade total, macro e microporosidade (mesa de tensão, com amostras saturadas) e composição granulométrica, pelo método da pipeta.

Os dados foram testados quanto à normalidade, para obtenção do intervalo de confiança para a média $(\alpha=0,01)($ Tabelas 1 e 2).

A cada dois meses, foi avaliado o incremento médio em altura, diâmetro do colo e área de copa, até os 10 meses, e a sobrevivência dos eucaliptos foi determinada aos quatro meses de idade.

Mensalmente, a partir do quinto mês após o plantio foi quantificada a clorofila (Valor SPAD), por meio do Medidor Portátil de Clorofila (MPC) (ARGENTA et al., 2001; ZOTARELLI et al., 2002; TORRES NETO et al., 2002; CHANG e ROBISON, 2003; CARVALHO et al., 2004; TORRES NETO et al., 2005; ALMEIDA et al., 2005) e a Massa Específica Foliar (MEF). Adicionalmente, foi testada a correlação entre o comprimento foliar e o MPC, em duas épocas (período chuvoso e seco).

A quantidade de clorofila foi avaliada em três plantas por parcela, com dimensões mais próximas às médias da parcela. A leitura foi efetuada na primeira folha expandida do sexto ramo a partir do ápice das plantas. Com o auxílio de um medidor portátil de clorofila (modelo SPAD-502, Minolta), procedeu-se a leitura em três pontos do limbo foliar, sendo utilizado para análise o valor médio obtido a partir destes.

R. Árvore, Viçosa-MG, v.33, n.1, p.67-79, 2009 
Tabela 1 - Caracterizações química e física do substrato da cava resultante da extração de argila, antes do plantio de eucalipto (média \pm intervalo de confiança)

Table 1 - Chemical and physical characterization of the digging substratum, resulting from the clay mining, before the eucalyptus planting

\begin{tabular}{|c|c|c|c|c|}
\hline \multirow{3}{*}{ Parâmetros } & \multicolumn{2}{|c|}{ Local sem a Sesbania virgata } & \multicolumn{2}{|c|}{ Local com a Sesbania virgata } \\
\hline & \multicolumn{4}{|c|}{ Profundidade $(\mathrm{cm})$} \\
\hline & $0-20$ & $20-40$ & $0-20$ & $20-40$ \\
\hline $\mathrm{Ph}\left(\mathrm{H}_{2} \mathrm{O}\right)$ & $5,7 \pm 0,2$ & $6,1 \pm 0,7$ & $5,7 \pm 0,5$ & $5,7 \pm 0,3$ \\
\hline $\mathrm{P}\left(\mathrm{mg} \mathrm{dm}^{-3}\right)$ & $26,4 \pm 33,2$ & $13,9 \pm 11,4$ & $18,6 \pm 16,0$ & $16,8 \pm 14,7$ \\
\hline $\mathrm{K}\left(\mathrm{cmol}_{\mathrm{c}} \mathrm{dm}^{-3}\right)$ & $0,2 \pm 0,0$ & $0,1 \pm 0,0$ & $0,1 \pm 0,0$ & $0,1 \pm 0,0$ \\
\hline $\mathrm{Ca}\left(\mathrm{cmol}_{\mathrm{c}} \mathrm{dm}^{-3}\right)$ & $8,0 \pm 3,5$ & $5,6 \pm 3,9$ & $7,3 \pm 2,2$ & $6,4 \pm 2,0$ \\
\hline $\operatorname{Mg}\left(\mathrm{cmol}_{\mathrm{c}}^{\mathrm{c}} \mathrm{dm}^{-3}\right)$ & $4,8 \pm 0,6$ & $3,3 \pm 1,8$ & $5,0 \pm 1,1$ & $4,6 \pm 0,2$ \\
\hline $\mathrm{H}+\mathrm{Al}\left(\mathrm{cmol}_{\mathrm{c}} \mathrm{dm}^{-3}\right)$ & $2,8 \pm 0,3$ & $1,9 \pm 1,1$ & $3,0 \pm 0,8$ & $3,0 \pm 0,6$ \\
\hline $\mathrm{Na}\left(\mathrm{cmol}_{\mathrm{c}} \mathrm{dm}^{-3}\right)$ & $1,2 \pm 0,3$ & $1,7 \pm 0,9$ & $1,2 \pm 1,2$ & $1,9 \pm 0,8$ \\
\hline $\mathrm{SB}\left(\mathrm{cmol}_{\mathrm{c}} \mathrm{dm}^{-3}\right)$ & $14,1 \pm 3,6$ & $10,6 \pm 5,2$ & $13,9 \pm 2,3$ & $13,1 \pm 1,9$ \\
\hline $\mathrm{T}\left(\mathrm{cmol}_{\mathrm{c}} \mathrm{dm}^{-3}\right)$ & $16,9 \pm 3,6$ & $12,5 \pm 6,2$ & $16,9 \pm 2,4$ & $15,9 \pm 1,6$ \\
\hline $\mathrm{MO}\left(\mathrm{g} \mathrm{dm}^{-3}\right)$ & $25,2 \pm 4,6$ & $16,0 \pm 10,7$ & $24,1 \pm 10,4$ & $22,0 \pm 6,7$ \\
\hline $\mathrm{V}(\%)$ & $83,5 \pm 4,0$ & $85,2 \pm 3,1$ & $82,5 \pm 4,4$ & $82,0 \pm 4,9$ \\
\hline $\mathrm{CE}_{\mathrm{es}}\left(\mathrm{dS} \mathrm{m}^{-1}\right)$ & $1,4 \pm 0,7$ & $1,5 \pm 1,5$ & $1,3 \pm 0,5$ & $1,5 \pm 0,4$ \\
\hline Areia $(\%)$ & $12,40 \pm 12,12$ & $34,17 \pm 50,39$ & $12,94 \pm 2,27$ & $12,19 \pm 2,25$ \\
\hline Silte (\%) & $37,07 \pm 3,66$ & $27,42 \pm 17,21$ & $39,64 \pm 2,63$ & $39,31 \pm 1,74$ \\
\hline Argila (\%) & $50,52 \pm 10,90$ & $38,41 \pm 33,20$ & $47,42 \pm 3,96$ & $48,49 \pm 3,34$ \\
\hline
\end{tabular}

MO: matéria orgânica; T= CTC pH 7,0; SB: soma de bases trocáveis, V: \%saturação de bases, $\mathrm{CE}_{\mathrm{es}}$ : condutividade elétrica da pasta saturada;

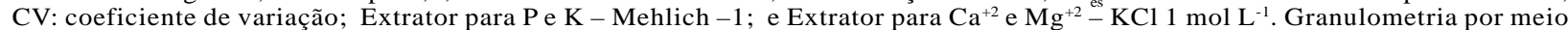
do método da pipeta.

Tabela 2 - Densidade do substrato $(\rho)$ e de partícula ( $\rho$ s), porosidade total (PT), macroporosidade (Mac.) e microporosidade (Mic.) do substrato da cava (média \pm intervalo de confiança)

Table 2 - Substratum density $(\rho)$ and of particle $(\rho s)$, total porosity (PT), macroporosity (Mac.) and microporosity (Mic.) of the digging clay mining substratum

\begin{tabular}{|c|c|c|c|c|c|}
\hline \multirow{3}{*}{ Prof. $(\mathrm{cm})$} & \multicolumn{5}{|c|}{ Local sem a presença de Sesbania virgata } \\
\hline & $\rho$ & $\rho s$ & Mac. & Mic. & $\mathrm{PT}$ \\
\hline & \multicolumn{2}{|c|}{$\left(\mathrm{Mg} \mathrm{m}^{-3}\right)$} & \multicolumn{3}{|c|}{$\left(\mathrm{m}^{3} \mathrm{~m}^{-3}\right)$} \\
\hline $0-10$ & $1,21 \pm 0,25$ & $2,58 \pm 0,08$ & $0,01 \pm 0,03$ & $0,53 \pm 0,10$ & $0,53 \pm 0,09$ \\
\hline $10-20$ & $1,22 \pm 0,10$ & $2,59 \pm 0,15$ & $0,00 \pm 0,01$ & $0,52 \pm 0,03$ & $0,53 \pm 0,03$ \\
\hline & & cal com a p & esbania vir & & \\
\hline \multirow[t]{2}{*}{ Prof. $(\mathrm{cm})$} & $\rho$ & ps & Mac. & Mic. & $\mathrm{P} \mathrm{T}$ \\
\hline & \multicolumn{2}{|c|}{$\left(\mathrm{Mg} \mathrm{m}^{-3}\right)$} & \multicolumn{3}{|c|}{$\left(\mathrm{m}^{3} \mathrm{~m}^{-3}\right)$} \\
\hline $0-10$ & $1,19 \pm 0,03$ & $2,47 \pm 0,07$ & $0,08 \pm 0,22$ & $0,44 \pm 0,20$ & $0,52 \pm 0,02$ \\
\hline $10-20$ & $1,24 \pm 0,10$ & $2,53 \pm 0,17$ & $0,12 \pm 0,18$ & $0,38 \pm 0,21$ & $0,50 \pm 0,05$ \\
\hline
\end{tabular}

A Massa Específica Foliar (MEF) foi determinada em uma planta de cada parcela, da qual foram coletadas quatro folhas (uma por quadrante), totalmente expandidas e localizadas no terço superior da planta. O critério de seleção das plantas foi o mesmo da determinação da quantidade de clorofila. Após a coleta, as folhas foram acondicionadas numa caixa de isopor com gelo, envoltas em filme plástico. Posteriormente, procedeuse à medição da área foliar (modelo LI-3000, LI-COR
Inc.). Em seguida, o material foi seco em estufa de ventilação forçada a $65^{\circ} \mathrm{C}$, por $48 \mathrm{~h}$, e a seguir, pesado.

Durante os meses de janeiro e julho de 2004 (6 e 12 meses após o plantio), na segunda folha a partir do ápice, das três plantas de cada parcela utilizadas para determinação do valor SPAD, foi realizada a medição, a cada três dias, do valor SPAD e comprimento foliar.

Com o objetivo de caracterizar o nível de

\section{R. Árvore, Viçosa-MG, v.33, n.1, p.67-79, 2009}


luminosidade a que as plantas estavam sendo submetidas no momento das avaliações de valor SPAD e MEF, foi determinado, com o auxílio de um "quantômetro em linha", o Fluxo de Fótons Fotossintéticos - FFF (Tabela 4). No período do dia próximo ao zênite, em dias não nublados, as leituras foram efetuadas num ponto central dentro de cada parcela a $1 \mathrm{~m}$ de altura.

Aos 12 meses de idade, foram avaliados a área superficial e o comprimento de raízes finas dos eucaliptos, nos dois sistemas de cultivo. As coletas foram realizadas com o auxílio de um trado $(\phi=2,5 \mathrm{~cm})$, na profundidade de 0 a $10 \mathrm{~cm}$. Foram tomadas duas amostras por repetição, sendo uma retirada a $20 \mathrm{~cm}$ e outra a $40 \mathrm{~cm}$ de distância da planta média, na linha de plantio do eucalipto. Posteriormente, procedeu-se a separação das raízes do solo mediante a lavagem.

A quantificação de raízes finas foi realizada por meio do programa QuantRoot ${ }^{2}$, que realiza medição direta das raízes nas imagens digitalizadas em escala real.

Foi realizada análise conjunta dos experimentos por sistema de cultivo. Para a análise dos incrementos foi utilizado o esquema fatorial 4 × 4 × 2 , sendo quatro espécies, quatro períodos de avaliação e dois sistemas de cultivo. Para o valor SPAD e MEF, o esquema fatorial foi $2 \times 4 \times 4$ e $2 \times 4 \times 7$, respectivamente. E cada valor correspondeu, em ordem, aos sistemas de plantio, número de espécies e períodos de avaliação.

Os dados foram testados quanto à homocedasticidade, pelo teste de Cochran, e quanto à distribuição normal dos resíduos, pelo teste de Lilliefors. Para a característica sobrevivência, constatouse heterocedasticidade, e foi realizada a transformação dos dados para arcosseno $(\% / 100)^{1 / 2}$.

Os dados foram submetidos à análise de variância, sendo constatado significância. Para as características quantitativas, foram ajustadas equações de regressão pelo método de regressão sequencial, sendo observada a distribuição gráfica dos resíduos padronizados. Para as características qualitativas, aplicou-se, em razão da sua variabilidade, o teste de Tukey (5\%) ou o de Duncan (5\%).

Tabela 3 - Composição granulométrica ${ }^{1}$ do substrato da cava de extração de argila (média \pm intervalo de confiança) Table 3 - Texture of the digging of clay mining substratum

\begin{tabular}{|c|c|c|c|}
\hline \multirow{3}{*}{ Prof. (cm) } & \multicolumn{3}{|c|}{ Local sem a presença de Sesbania virgata } \\
\hline & Areia & Silte & Argila \\
\hline & \multicolumn{3}{|c|}{$(\%)$} \\
\hline $0-20$ & $12,40 \pm 12,12$ & $37,07 \pm 3,66$ & $50,52 \pm 10,90$ \\
\hline \multirow[t]{2}{*}{$20-40$} & $34,17 \pm 50,39$ & $27,42 \pm 17,21$ & $38,41 \pm 33,20$ \\
\hline & \multicolumn{3}{|c|}{ Local com a presença de Sesbania virgata } \\
\hline \multirow[t]{2}{*}{ Prof. (cm) } & Areia & Silte & Argila \\
\hline & \multicolumn{3}{|c|}{$(\%)$} \\
\hline $0-20$ & $12,94 \pm 2,27$ & $39,64 \pm 2,63$ & $47,42 \pm 3,96$ \\
\hline $20-40$ & $12,19 \pm 2,25$ & $39,31 \pm 1,74$ & $48,49 \pm 3,34$ \\
\hline
\end{tabular}

${ }^{1}$ Determinada pelo método da pipeta.

Tabela 4 - Fluxo de fótons fotossintéticos (média \pm intervalo de confiança) no monocultivo e no plantio consorciado durante o período experimental, em uma cava de extração de argila

Table 4 - Photosynthetic Active Radiation in the pure and mixed planting in experimental period, in a clay mining digging

\begin{tabular}{cccc}
\hline Meses após o plantio dos eucaliptos & Mês & Monocultivo & $\mathrm{mmol}^{-2} \cdot \mathrm{s}^{-1}$ \\
\cline { 2 - 4 } & & $2000 \pm 0,0$ & $1183,5 \pm 494,5$ \\
6 & Dez/2003 & $2000 \pm 0,0$ & $1679,1 \pm 324,7$ \\
7 & Jan/2004 & $2000 \pm 0,0$ & $1761,1 \pm 238,6$ \\
8 & Fev/2004 & $2000 \pm 0,0$ & $1070,3 \pm 199,7$ \\
9 & Março/2004 & $1059,3 \pm 35,7$ & $83,5 \pm 134,2$ \\
10 & Abril/2004 & $1074 \pm 0,0$ & $89,4 \pm 140,1$ \\
11 & Maio/2004 & $1010 \pm 0,0$ & $85,6 \pm 174,4$ \\
\hline
\end{tabular}

${ }^{2}$ Prof Elpídio Inácio Fernandes Filho, UFV. 


\section{RESULTADOS E DISCUSSÃO}

Os atributos químicos do substrato (Tabela 1) encontram-se em níveis elevados ou adequados para a implantação do eucalipto, exceto $\mathrm{P}$ assimilável (BARROS et al., 2000; NOVAIS et al., 1986). No entanto, para alguns atributos físicos como macroporosidade, os valores determinados foram muito baixos (Tabela 2), com valores próximos aos encontrados por Valicheski et al. (2003) em outras cavas de extração de argila

A sobrevivência das espécies (Tabela 5) tanto no plantio consorciado quanto no monocultivo pode ser considerada satisfatória, se for tomado como exemplo um ensaio de diferentes procedências de eucalipto e sistemas de plantio em solos de tabuleiro da região, onde o percentual médio de sobrevivência de Eucalyptus camaldulensis e E. pellita foi de $75 \%$ (BARROSO e CARNEIRO, 2002).

Não houve diferença de sobrevivência entre as espécies. Entretanto, em razão do sistema de plantio, foi observada sobrevivência $8,74 \%$ maior no consorciado. Piotto et al. (2004), estudando plantios mistos na Costa Rica, também constataram maior sobrevivência de espécies como Schizolobium parahyba (Vell.) Blake em consórcio que em monocultivos, com diferença de até $40 \%$.

Tabela 5 - Taxas de sobrevivência de espécies de eucalipto, aos quatro meses de idade, no monocultivo e no plantio consorciado com Sesbania virgata

Table 5 - Survival of eucalyptus species, to the 4 months after planting, in pure system and mixed with Sesbania virgata

\begin{tabular}{lccc}
\hline Espécie & \multicolumn{2}{c}{ Sobrevivência } \\
\cline { 2 - 3 } & Monocultivo & $\begin{array}{c}\text { Plantio } \\
\text { consorciado }\end{array}$ \\
\cline { 2 - 2 } $\begin{array}{c}\text { Dados } \\
\text { orig. }(\%) *\end{array}$ & $\begin{array}{c}\text { Dados }(\%) * \\
\text { orig. }\end{array}$ \\
\hline Eucalyptus camaldulensis & $90,28 \mathrm{a}$ & \\
Eucalyptus tereticornis & $97,22 \mathrm{a}$ & & $100,00 \mathrm{a}$ \\
Eucalyptus robusta & $83,33 \mathrm{a}$ & & $100,00 \mathrm{a}$ \\
Eucalyptus pellita & $91,67 \mathrm{a}$ & $98,61 \mathrm{a}$ \\
\hline Média & $90,63 \mathrm{~B}$ & $99,31 \mathrm{~A}$ \\
\hline CV(\%) & \multicolumn{3}{c}{8,63} \\
\hline
\end{tabular}

Médias seguidas por letras iguais maiúsculas na linha e minúsculas na coluna não diferem entre si, pelo teste de Tukey $(5 \%)$. * Análise realizada com dados transformados em $\operatorname{arcosseno}(\%)$ $100) .^{1 / 2}$

R. Árvore, Viçosa-MG, v.33, n.1, p.67-79, 2009
O favorecimento da sobrevivência das plantas no plantio consorciado pode ter ocorrido em razão das condições favoráveis promovidas pela sesbânia às plantas de eucalipto, mantendo a umidade do substrato e, ou, protegendo-as contra intempéries ambientais, como vento e alta radiação solar (Tabela 4).

As interações entre as espécies em plantios consorciados nem sempre são, entretanto, positivas. Jesus e Dias (1988), estudando monocultivos e consórcios de Eucalyptus urophylla S. T. Blake e Leucaena leucocephala (Lam), no espaçamento 2,73 x 2,73 m, observaram, aos 7 anos, maior sobrevivência do eucalipto no monocultivo. Apesar do espaçamento por eles utilizado ser um pouco mais amplo do que neste ensaio, a menor sobrevivência do eucalipto consorciado parece estar associada à característica mais agressiva da leucena, já mencionada por Mafra et al. (1998b) em outro ensaio.

Também não foram observadas diferenças entre as espécies para diâmetro do colo, altura e área de copa no $10^{\circ}$ mês após o plantio (Tabela 6).

O monocultivo constou plantas com diâmetro do colo e área de copa superiores às do consórcio em 15,97\% e 20,97\%, respectivamente (Tabela 6). Não houve diferença de altura entre as espécies, nem variação dessa característica entre os plantios. Também, Barroso e Carneiro (2002), em solos de tabuleiro da região, com monocultivos de diferentes espécies e procedências de eucalipto (Eucalyptus grandis, E. camaldulensis, E. pellita, E. urophylla, Corymbia citriodora) e em consórcio de E. camaldulensis com Tectona grandis e Acacia mangium, não encontraram efeito dos sistemas de plantio na altura dos eucaliptos aos 6 meses e 1 ano de idade.

Neste ensaio, os eucaliptos estavam com altura média, aos 12 meses de idade, de 2,81 m. Nessa fase, o E. camaldulensis, quando consorciado com a leguminosa Acacia mangium, apresentou 3,99 m de altura. Esses valores se encontraram próximos ou inferiores aos encontrados nessa cava em plantios com 10 meses de idade.

Os maiores valores de diâmetro do colo e área de copa dos eucaliptos no monocultivo indicam possível competição inicial entre as espécies quando consorciadas, principalmente porque a sesbânia já estava implantada antes do plantio dos eucaliptos. É possível que, com o crescimento das espécies de eucalipto, essas diferenças dendrométricas entre os sistemas de plantio sejam alteradas de forma gradativa. 
Tabela 6 - Diâmetro do colo, altura e área de copa das espécies de eucalipto aos 10 meses de idade, no monocultivo e no plantio consorciado com Sesbania virgata

Table 6 - Collar diameter, height and canopy area, of the eucalyptus species, to the 10 months after planting, in pure and mixed system with Sesbania virgata

\begin{tabular}{|c|c|c|c|c|c|c|}
\hline \multirow[t]{2}{*}{ Espécie } & \multicolumn{2}{|c|}{ Diâmetro do colo (mm) } & \multicolumn{2}{|c|}{ Altura (m) } & \multicolumn{2}{|c|}{ Área de copa $\left(\mathrm{m}^{2}\right)$} \\
\hline & Monocultivo & Consórcio & Monocultivo & Consórcio & Monocultivo & Consórcio \\
\hline E. camaldulensis & 43,47 a & $36,07 \mathrm{a}$ & 3,42 a & $3,40 \mathrm{a}$ & $2,20 \mathrm{a}$ & 1,96 a \\
\hline E. tereticornis & 51,23 a & 41,68 a & 3,47 a & $3,17 \mathrm{a}$ & $2,85 \mathrm{a}$ & $1,84 \mathrm{a}$ \\
\hline E. robusta & $44,10 \mathrm{a}$ & $41,34 \mathrm{a}$ & 2,92 a & 3,43 a & $2,77 \mathrm{a}$ & $2,60 \mathrm{a}$ \\
\hline E. pellita & $46,34 \mathrm{a}$ & 43,02 a & $2,84 \mathrm{a}$ & $3,26 \mathrm{a}$ & $2,94 \mathrm{a}$ & $2,25 \mathrm{a}$ \\
\hline Média & $46,29 \mathrm{~A}$ & $40,53 \mathrm{~B}$ & $3,16 \mathrm{~A}$ & $3,31 \mathrm{~A}$ & $2,69 \mathrm{~A}$ & $2,16 \mathrm{~B}$ \\
\hline$\overline{\mathrm{CV}(\%)}$ & \multicolumn{2}{|c|}{14,24} & \multicolumn{2}{|c|}{11.65} & \multicolumn{2}{|c|}{23,96} \\
\hline
\end{tabular}

Para a mesma característica, médias seguidas por letras iguais maiúsculas na linha e minúsculas na coluna não diferem entre si, pelo teste de Tukey $(5 \%)$.

A diferença no comportamento dos eucaliptos nos dois sistemas de plantio pode ser observada na Figura 2, para incremento em diâmetro do colo na Figura 3 e para incremento em altura e na Figura 4, para incrementos em área de copa. Observou-se que, da mesma forma da análise aos 10 meses de idade, houve efeito dos sistemas de plantio nos incrementos em diâmetro do colo e área de copa.

Embora não tenham sido detectadas diferenças de altura entre as espécies, nem entre o comportamento destas diante do sistema de plantio (Tabela 6), ao longo do tempo foi observado padrão de incremento diferenciado das espécies, conforme o sistema de plantio adotado (Figura 3). Essas diferenças, em especial no plantio consorciado, parecem estar associadas ao manejo adotado e à sensibilidade delas em resposta a esse manejo.

No período compreendido entre o $8^{\circ}$ e o $10^{\circ}$ mês houve incremento mais acentuado em altura das espécies no plantio consorciado, exceto em E. tereticornis, que apresentou incremento linear (Figura 3A). Nos meses antecedentes ( $6^{\circ}$ ao $8^{\circ}$ mês $)$, houve queda nesse incremento.

No período compreendido entre o $6^{\circ}$ e o $10^{\circ}$ mês, ocorreu de forma sequencial queda na disponibilidade hídrica, do $6^{\circ}$ para o $8^{\circ}$ mês (Figura 1 ), seguida de aumento e maior sombreamento pela interceptação das copas das sesbânias (Tabela 4). O incremento em altura nessa ocasião, entre o $8^{\circ}$ e o $10^{\circ}$ mês, foi mais expressivo em E. robusta $\mathrm{Sm}$ e E. pellita $\mathrm{F}$. Muell. - espécies essas que apresentaram maior valor SPAD (Tabela 7).

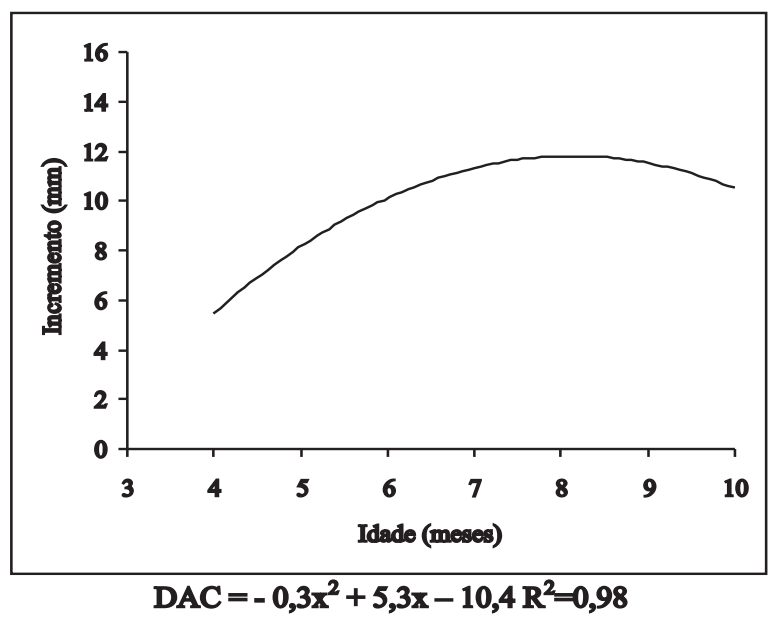

Figura 2 - Incremento médio em diâmetro do colo, ao longo do tempo, de espécies de eucalipto no plantio consorciado (A) e no monocultivo (B).

Figure 2 -Diameter at soil level increment, along the time, of eucalyptus species in the mixed (A) and in the pure planting $(B)$.

Apesar de o valor SPAD, na análise ao longo do tempo, não ter variado entre as espécies e sistemas de plantio (Figura 5), essa característica, por ser uma estimativa de teor de clorofila, com papel fundamental na aquisição de carbono, pode ter colaborado com o aumento mais acentuado do incremento em altura de E. robusta Sm e E. pellita F. Muell, durante o período de maior interceptação de luz pela sesbânias. Assim, espera-se que essa estimativa se correlacione com algumas características de crescimento das espécies. Nesse raciocínio, procurou-se correlacionar os valores SPAD com o comprimento foliar das espécies de eucalipto em períodos chuvoso e seco.

R. Árvore, Viçosa-MG, v.33, n.1, p.67-79, 2009 


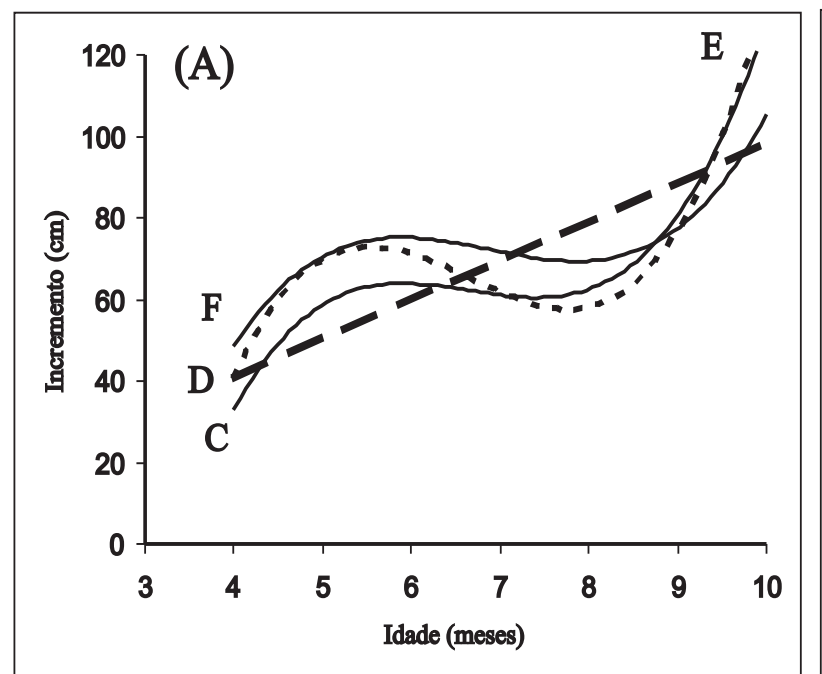

C $-E$. pellita $=2,1 \mathrm{x}^{3}-41,1 \mathrm{x}^{2}+269,6 \mathrm{x}-520,3 \mathrm{R}^{2}=1$

D $-E$. tereticornis $=9,6 \mathrm{x}+2,2 \mathrm{r}^{2}=0,99$

$\mathrm{E}-E$. robusta $=2,8 \mathrm{x}^{3}-55,6 \mathrm{x}^{2}+359,7 \mathrm{x}-686,8 \mathrm{R}^{2}=1$

$\mathrm{F}-\mathrm{E}$. camaldulensis $=1,5 \mathrm{x}^{3}-31,9 \mathrm{x}^{2}+214,7 \mathrm{x}-399,5 \mathrm{R}^{2}=1$

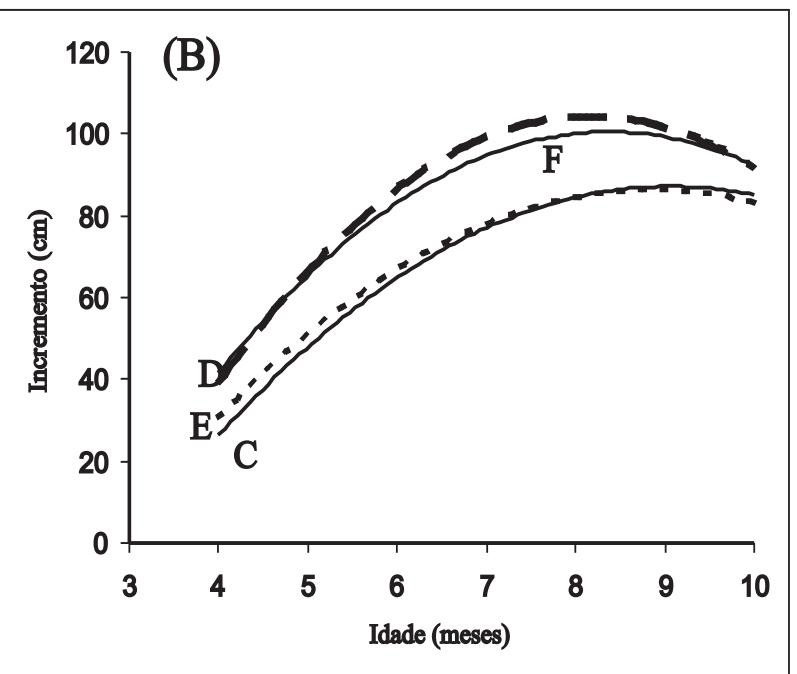

C - E. pellita $=-2,4 \mathrm{x}^{2}+43 \mathrm{x}-107,9 \mathrm{R}^{2}=0,99$

D - E. tereticornis $=-3,8 \mathrm{x}^{2}+61,3 \mathrm{x}-146 \mathrm{R}^{2}=0,99$

$\mathrm{E}-\mathrm{E}$. robusta $=-2,3 \mathrm{x}^{2}+41,6 \mathrm{x}-98,6 \mathrm{R}^{2}=0,98$

$\mathrm{F}-E$. camaldulensis $=-3,1 \mathrm{x}^{2}+51,8 \mathrm{x}-116 \mathrm{R}^{2}=0,99$

Figura 3 - Incremento médio em altura, ao longo do tempo, de espécies de eucalipto, no plantio consorciado (A) e no monocultivo (B).

Figure 3 -Height increment, along the time, of eucalyptus species in the mixed (A) and in the pure planting (B).

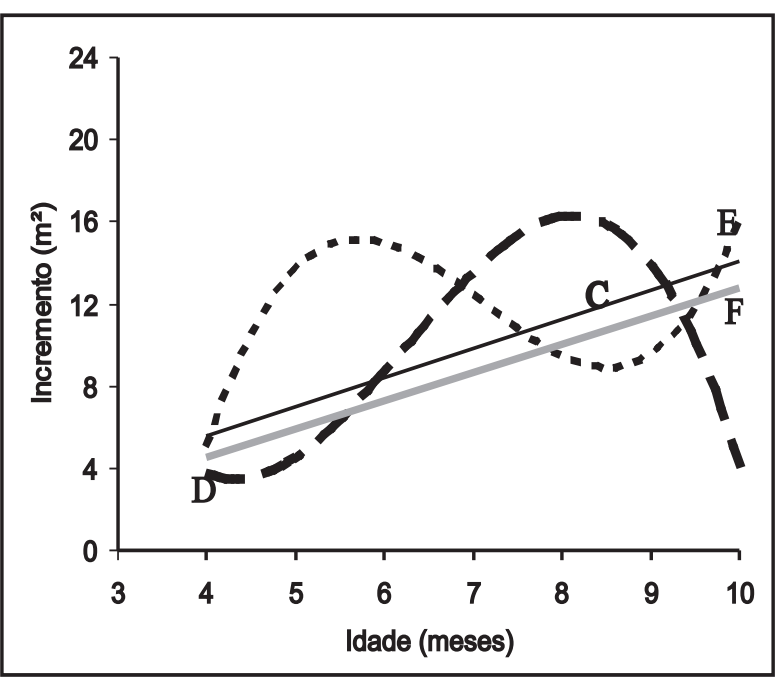

C - E. pellita $=2,1 \mathrm{x}-3,1 \mathrm{r}^{2}=0,88$

$\mathrm{D}-\mathrm{E}$. tereticornis $=-0,3 \mathrm{x}^{3}+5,8 \mathrm{x}^{2}-29,6 \mathrm{x}+49,6 \mathrm{R}^{2}=0,99$

$\mathrm{E}-\mathrm{E}$. robusta $=0,5 \mathrm{x}^{3}-10,3 \mathrm{x}^{2}+71,1 \mathrm{x}-146,5 \mathrm{R}^{2}=1$

$\mathrm{F}-\mathrm{E}$. camaldulensis $=1,6 \mathrm{x}-1,9 \mathrm{r}^{2}=0,83$

Figura 4 - Incremento em área de copa, ao longo do tempo, de espécies de eucalipto em dois sistemas de plantio.

Figure 4-Canopy area increment, along the time, of eucalyptus species in the two plantings systems.
Tabela 7 - Médias referentes ao valor SPAD das espécies de eucalipto, em dois sistemas de plantio, tomados mensalmente do quinto ao oitavo mês

Table 7-SPAD value of the eucalyptus species, in two planting systems, taken monthly from the fifth to the eighth month

\begin{tabular}{lcc}
\hline Espécies & \multicolumn{2}{c}{ Valor SPAD } \\
\cline { 2 - 3 } & Monocultivo & Plantio consorciado \\
\hline E. camaldulensis & $26,44 \mathrm{c}$ & $29,56 \mathrm{~b}$ \\
E. tereticornis & $25,77 \mathrm{c}$ & $28,41 \mathrm{~b}$ \\
E. robusta & $40,26 \mathrm{a}$ & $38,45 \mathrm{a}$ \\
E. pellita & $34,01 \mathrm{~b}$ & $36,71 \mathrm{a}$ \\
\hline Média & $31,62 \mathrm{~A}$ & $33,28 \mathrm{~A}$ \\
\hline CV $(\%)$ & \multicolumn{3}{c}{12,47} \\
\hline
\end{tabular}

Para a mesma característica, médias seguidas por letras iguais maiúsculas na linha e minúscula na coluna não diferem entre si, pelo teste de Tukey (5\%).

O valor SPAD correlacionou-se positivamente com o comprimento foliar nas duas épocas de avaliação (Tabela 8), indicando relação estreita entre o valor obtido no medidor portátil de clorofila (MPC) e no crescimento foliar. 


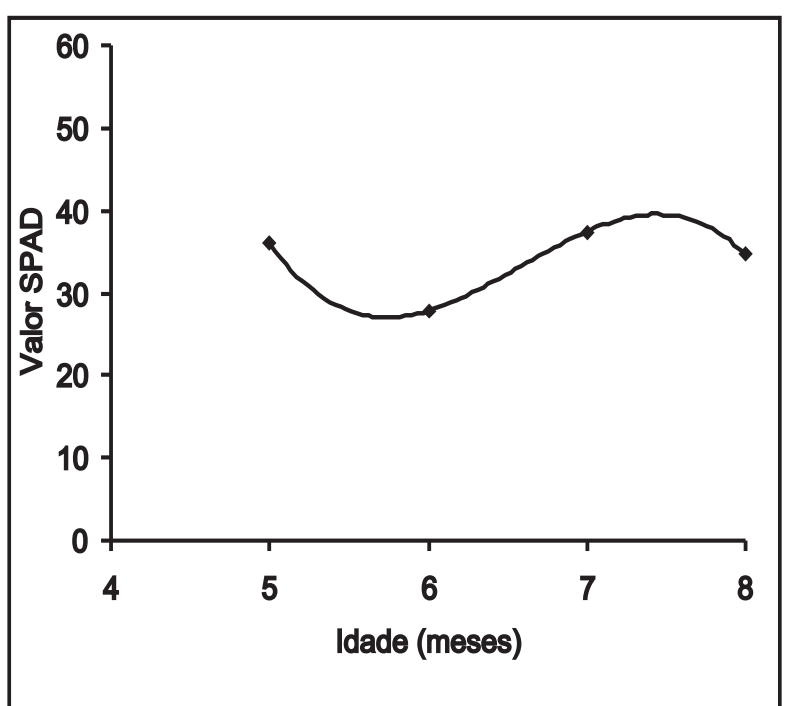

$\mathrm{SPAD}=-5,9 \mathrm{x}^{3}+117,2 \mathrm{x}^{2}-763,8 \mathrm{x}+1661,9 \mathrm{R}^{2}=1$

Figura 5 - Valor SPAD médio de espécies de eucalipto. Figure 5 - SPAD value of the eucalyptus species.

Detectou-se efeito dos sistemas de plantio e da época de avaliação da Massa Específica Foliar (MEF), bem como comportamento diferenciado entre as espécies (Figura 6). A MEF, razão entre a massa e a área foliar, denota "espessura" de folha e é uma característica fisiológica muito influenciada pela incidência de luz (TAIZ e ZEIGER, 2004).

No monocultivo, as curvas que descrevem os valores da MEF são das quatro espécies de eucalipto, lineares ao longo do tempo (Figura 6B). No plantio consorciado, em que a competição inicial por luz, água e nutrientes possivelmente foi maior, as oscilações nos valores de MEF ocorridas ao longo do tempo indicaram que as variações de luz e água influenciaram grandemente essa característica (Figura 6A).
Todas as espécies de eucalipto, no plantio consorciado apresentaram, para a característica MEF, queda acentuada entre o $8^{\circ}$ e o $9^{\circ}$ mês, seguida de um aumento até meados do mês seguinte. A queda na MEF no consórcio coincidiu com o menor Fluxo de Fótons Fotossintéticos (FFF) no sub-bosque da sesbânia (Tabela 4) e diminuição da precipitação pluviométrica, mês de março de 2004 (Figura 1), podendo o aumento estar associado à maior disponibilidade luminosa, proporcionada pela poda na leguminosa realizada 15 dias antes da avaliação ao $10^{\circ}$ mês e de água.

O incremento em altura de E. tereticornis no consórcio foi linear ao longo do tempo, ainda que inferior ao plantio puro (Figura 3), demonstrando menor influência do consórcio e do manejo via podas periódicas sobre essa espécie para essa característica. Isso pode ser explicado se se observar a sua produção em área superficial e comprimento de raízes finas no plantio consorciado (Tabela 9).

A oscilação no incremento em altura dos eucaliptos ao longo do tempo, no plantio consorciado, conforme discutido anteriormente, parece ser função da disponibilidade hídrica e de luz. Entre o $8^{\circ}$ e o $10^{\circ}$ mês houve aumento na precipitação pluviométrica, associado à maior disponibilidade de luz proporcionada pela poda. Nesse período, todas as espécies, exceto o E. tereticornis, responderam no incremento em altura, notadamente as espécies com maior valor SPAD (E. robusta e $E$. pellita). É possível que a falta de resposta do $E$. tereticornis esteja associado à sua menor produção de raízes finas. Como essas raízes são importantes no acesso aos recursos do solo (GONÇALVES, 1995; GRANT et al., 2001; FREITAS et al., 2008), quando os fatores que poderiam estar limitando são minimizados, as espécies com sistema radicular mais estabelecido respondem rapidamente em crescimento.

Tabela 8 - Coeficiente de correlação de Pearson entre o valor SPAD e o comprimento foliar das espécies de eucalipto nos períodos chuvoso e seco, nos dois sistemas de plantio

Table 8 - Pearson Correlation Coefficient among the SPAD value and leaflength of the eucalyptus species in the rainy and dry period, in the two planting systems

\begin{tabular}{|c|c|c|c|c|}
\hline \multirow[t]{3}{*}{ Espécie } & \multicolumn{2}{|c|}{ Monocultivo } & \multicolumn{2}{|c|}{ Plantio consorciado } \\
\hline & Chuvoso & Seco & Chuvoso & Seco \\
\hline & $\mathrm{r}$ & $\mathrm{r}$ & $\mathrm{r}$ & $\mathrm{r}$ \\
\hline E. camaldulensis & $0,66 * *$ & $0,87 * *$ & $0,73 * *$ & $0,45 * *$ \\
\hline E. tereticornis & $0,80 * *$ & $0,90 * *$ & $0,76 * *$ & $0,52 * *$ \\
\hline E. robusta & $0,53 * *$ & $0,71 * *$ & $0,68 * *$ & $0,30 * *$ \\
\hline E. pellita & $0,80 * *$ & $0,79 * *$ & $0,65 * *$ & $0,87 * *$ \\
\hline
\end{tabular}

** Teste $\mathrm{t}$ - significância a $1 \%$. 


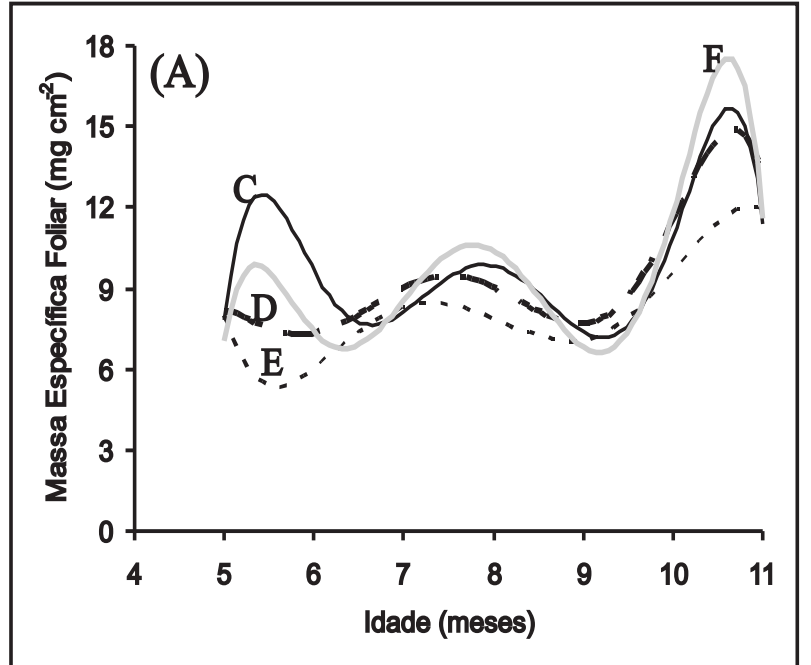

C-E. pellita $=-0,1 \mathrm{x}^{6}+5 \mathrm{x}^{5}-90 \mathrm{x}^{4}+900 \mathrm{x}^{3}-5300 \mathrm{x}^{2}+15900 \mathrm{x}-19600 \mathrm{R}^{2}=1$

D-E. tereticornis $=-0,03 \mathrm{x}^{6}+\mathrm{x}^{5}-30 \mathrm{x}^{4}+300 \mathrm{x}^{3}-1400 \mathrm{x}^{2}+3900 \mathrm{x}-4500 \mathrm{R}^{2}=1$

E-E. robusta $=-0,07 \mathrm{x}^{5}+3 \mathrm{x}^{4}-40 \mathrm{x}^{3}+300 \mathrm{x}^{2}-1300 \mathrm{x}+2000 \mathrm{R}^{2}=0,93$

F-E. camaldulensis $=-0,1 \mathrm{x}^{6}+5 \mathrm{x}^{5}-90 \mathrm{x}^{4}+900 \mathrm{x}^{3}-5400 \mathrm{x}^{2}+15900 \mathrm{x}-19300 \mathrm{R}^{2}=1$

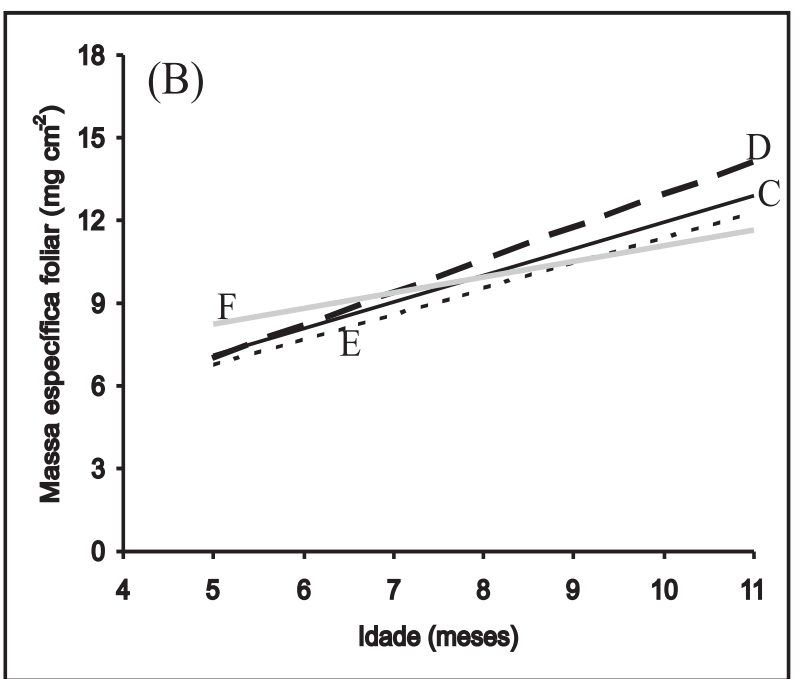

C - E. pellita $=\mathrm{x}+2 \mathrm{r}^{2}=0,91$

D - E. tereticornis $=\mathrm{x}+1 \mathrm{r}^{2}=0,98$

$\mathrm{E}-$ E. robusta $=0,9 \mathrm{x}+2 \mathrm{r}^{2}=0,93$

$\mathrm{F}-$ E. camaldulensis $=0,6 \mathrm{x}+5 \mathrm{r}^{2}=0,79$

Figura 6 - Massa específica foliar média, ao longo do tempo, de espécies de eucalipto, no plantio consorciado (A) e no monocultivo (B).

Figure 6 - Specific Foliate Weight, along the time, of eucalyptus species in the mixed (A) and in the pure planting (B).

Tabela 9 - Raízes finas (diâmetro < 0,4 mm) produzidas por espécies de eucalipto, coletadas aos 12 meses de idade a 20 e $40 \mathrm{~cm}$ de distância da planta média de cada parcela, na profundidade de 0 a $10 \mathrm{~cm}$, no monocultivo e no plantio consorciado com Sesbania virgata

Table 9 - Fine roots $(<0,4 \mathrm{~mm})$ produced by eucalyptus species, collected at 12 months after the planting, distanced 20 and 40 of the medium plant for plot, in the depth from 0 to $10 \mathrm{~cm}$, in pure planting and associated with Sesbania virgata

\begin{tabular}{|c|c|c|c|c|}
\hline \multirow{3}{*}{ Espécie } & \multicolumn{4}{|c|}{ Distância $20 \mathrm{~cm}$} \\
\hline & \multicolumn{2}{|c|}{ Área Superficial $\left(\mathrm{cm}^{2} \mathrm{dm}^{-3}\right)$} & \multicolumn{2}{|c|}{ Comprimento $\left(\mathrm{m} \mathrm{dm}^{-3}\right)$} \\
\hline & Monocultivo & Consórcio & Monocultivo & Consórcio \\
\hline E. camaldulensis & $59,69 \mathrm{~A} \mathrm{a}$ & $17,52 \mathrm{~B} \mathrm{a}$ & $5,54 \mathrm{~A} \mathrm{ab}$ & $1,60 \mathrm{~B} \mathrm{a}$ \\
\hline E. pellita & $8,96 \mathrm{~A} \mathrm{~b}$ & $29,34 \mathrm{~A}$ a & $0,86 \mathrm{~A} \mathrm{c}$ & $2,22 \mathrm{~A} \mathrm{a}$ \\
\hline E. robusta & $82,51 \mathrm{~A}$ a & $27,71 \mathrm{~B}$ a & $6,40 \mathrm{~A} \mathrm{a}$ & $2,67 \mathrm{~B} \mathrm{a}$ \\
\hline E. tereticornis & $24,04 \mathrm{~A} \mathrm{~b}$ & $30,76 \mathrm{~A} \mathrm{a}$ & $2,43 \mathrm{~A} \mathrm{bc}$ & $3,09 \mathrm{~A} \mathrm{a}$ \\
\hline Média & 43,80 & 26,28 & 3,55 & 2,39 \\
\hline \multirow[t]{2}{*}{$\mathrm{CV}(\%)$} & \multicolumn{2}{|c|}{60,64} & \multicolumn{2}{|c|}{47,79} \\
\hline & \multicolumn{4}{|c|}{ Distância $40 \mathrm{~cm}$} \\
\hline \multirow[t]{2}{*}{ Espécie } & \multicolumn{2}{|c|}{ Área Superficial $\left(\mathrm{cm}^{2} \mathrm{dm}^{-3}\right)$} & \multicolumn{2}{|c|}{ Comprimento $\left(\mathrm{m} \mathrm{dm}^{-3}\right)$} \\
\hline & Monocultivo & Consórcio & Monocultivo & Consórcio \\
\hline E. camaldulensis & $65,60 \mathrm{~A} \mathrm{a}$ & $35,45 \mathrm{~B} \mathrm{ab}$ & $6,34 \mathrm{~A}$ a & $2,64 \mathrm{~B} \mathrm{ab}$ \\
\hline E. pellita & $24,04 \mathrm{~A} \mathrm{~b}$ & $27,30 \mathrm{~A} \mathrm{ab}$ & $1,96 \mathrm{~A} \mathrm{~b}$ & $2,67 \mathrm{~A} \mathrm{ab}$ \\
\hline E. robusta & $25,46 \mathrm{~A} \mathrm{~b}$ & $49,91 \mathrm{~A}$ a & $2,44 \mathrm{~A} \mathrm{~b}$ & $5,04 \mathrm{~A} \mathrm{a}$ \\
\hline E.tereticornis & $43,60 \mathrm{~A} \mathrm{ab}$ & $16,30 \mathrm{~A} \mathrm{~b}$ & $3,19 \mathrm{~A} \mathrm{~b}$ & $1,44 \mathrm{~A} \mathrm{~b}$ \\
\hline Média & 39,52 & 32,19 & 3,48 & 2,95 \\
\hline $\mathrm{CV}(\%)$ & \multicolumn{2}{|c|}{52,47} & \multicolumn{2}{|c|}{56,88} \\
\hline
\end{tabular}

Para a mesma característica, médias seguidas por letras iguais maiúsculas na linha e minúsculas na coluna não diferem entre si, pelo teste de Duncan $(5 \%)$.

R. Árvore, Viçosa-MG, v.33, n.1, p.67-79, 2009 
Apesar de esses resultados serem preliminares em um ensaio com vistas a respostas de médio e longo prazos, observou-se que podas sincronizadas em períodos de maior disponibilidade de água podem favorecer o incremento inicial em altura das espécies de eucalipto em sistemas consorciados.

A adoção de espaçamentos maiores pode vir a favorecer a produção do eucalipto consorciado com sesbânia em cavas de extração de argila, conforme observado por Vezzani et al. (2001), no consórcio de E. saligna (Smith) com Acacia mearnsii (Wild.), havendo redução do efeito da competição inicial e potencializando os benefícios da leguminosa no aporte de recursos. Esses benefícios poderão ser ainda observados nas avaliações ao longo do ciclo de produção do eucalipto.

Outro fator a ser considerado em consórcios é a ocorrência de pragas e doenças. No $6^{\circ}$ mês após o plantio dos eucaliptos, foi observada a presença de um coleóptero, ainda em processo de identificação, e sua ocorrência foi de 23,26\% nas espécies sob consórcio, com menor ocorrência no E. tereticornis $\operatorname{Sm}(15,28 \%)$. Não se observou presença do coleóptero no monocultivo.

\section{CONCLUSÕES}

Os plantios consorciados com Sesbania virgata (Cav.) Pers favoreceram a sobrevivência das espécies de eucalipto.

Os eucaliptos no monocultivo apresentaram maior crescimento inicial em diâmetro do colo e em área de copa, em relação ao plantio consorciado.

As espécies de eucalipto, nos primeiros 10 meses, são responsivas ao consórcio e ao manejo via podas periódicas, exceto o Eucalyptus tereticornis Sm.

\section{REFERÊNCIAS}

AMADOR, D. B.; VIANA, V. M. Sistemas agroflorestais para recuperação de fragmentos florestais. Série Técnica IPEF, v.12, n.32, p.105-110, 1998.

ALMEIDA, S. M. Z. et al. Alterações morfológicas e alocação de biomassa em plantas jovens de espécies florestais sob diferentes condições de sombreamento. Ciência Rural, v.35, n.1, p.62-68, 2005.

ARATO, H. D.; MARTINS, S. V.; FERRARI, S. H. S. Produção e decomposição de serrapilheira em um sistema agroflorestal implantado para recuperação de área degradada em Viçosa, $\mathrm{MG}$. Revista Árvore, v.27, n.5, p.715-721, 2003.
ARGENTA, G.; SILVA, P. R. F.; BORTOLINI, C. G. Clorofila na folha como indicador do nível de nitrogênio em cereais. Ciência Rural, v.31, n. 4 , p.715-722, 2001.

BALIEIRO, F. C.; FRANCO, A. A.; PEREIRA, M. G. Contribution of litter and nitrogen to soil under Pseudosamanea guachapele and Eucalyptus grandis plantations. Pesquisa Agropecuária Brasileira, v.39, n.6, p.597-601, 2004.

BARROS, N. F.; NEVES, J. C. L.; NOVAIS, R. F. Recomendação de fertilizantes minerais em plantios de eucalipto. In: GONÇALVES, J. L. M.;

BENEDETTI, V. (Eds.) Nutrição e fertilização florestal. Piracicaba: IPEF, 2000. p.269-286.

CARVALHO, J. G. et al. Uso do clorofilômetro Minolta SPAD-502 na predição do estado nutricional do algodoeiro herbáceo a nitrogênio e enxofre. FERTIBIO 2004, Lages. Anais... Lages, 2004. CD-ROM.

CECCON, E. et al. Consórcio entre Eucalyptus camaldulensis Dehn. Aos três anos de idade com diferentes cultivares de feijão (Phaseolus vulgaris L.). Revista Árvore, v.23, n.1, p.9-14, 1999.

CHANG, S. X.; ROBISON, D. J. Nondestructive and rapid estimation of hardwood foliar nitrogen status the SPAD-502 chlorophyll meter Forest Ecology and Management, v.6215, p.1-8, 2003.

COUTINHO, M. P. et al. Crescimento de mudas de Sesbania virgata (Cav.) Pers. plantadas em uma área degradada por extração de argila.Floresta, v.35, n.2, p.231-239, 2005.

COUTINHO, M. P. Crescimento de Sesbania virgata (Cav.) Pers. em cavas de extração de argila. 2003. 54f. Dissertação (Mestrado em Produção Vegetal) - Universidade Estadual do Norte Fluminense - Campos dos Goytacazes, 2003.

COSTA JÚNIOR, P. F. Comportamento de leguminosas arbóreas inoculadas com fungos micorrízicos arbusculares e rizóbio em estéril de extração de argila. 1997. 71f. Dissertação (Mestrado em Produção Vegetal) - Universidade Estadual do Norte Fluminense, Campos dos Goytacazes, 1997.

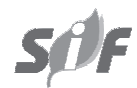

R. Árvore, Viçosa-MG, v.33, n.1, p.67-79, 2009 
DIAS, L. E. Caracterização de substratos para fins de recuperação de áreas degradadas. In: DIAS, L. E.; MELLO, J. W. V. (Eds.) Recuperação de áreas degradadas. Viçosa, MG: Sociedade Brasileira de Recuperação de Áreas Degradadas, 1998. 251p.

DRUMOND, M. A.; OLIVEIRA, V. R.; CARVALHO, O. M. Comportamento silvicultural de espécies e procedências de Eucalyptus na região dos tabuleiros costeiros do estado de Sergipe.

Revista Árvore, v.22, n.1, p.137-142, 1998.

FREITAS, T. A. S.; BARROSO, D. G.; CARNEIRO, J. G. Dinâmica de raízes de espécies arbóreas: Visão da Literatura. Ciência Florestal, v.18, n.1, p.133-142, 2008.

GONÇALVES, J. L. M. Sistema radicular de absorção do Eucalyptus grandis sob diferentes condições edáficas. Publicação da

Sociedade Brasileira de Silvicultura SBS. O Caminho da Certificação Florestal. v.17, n.61, 1995.

GRANT, C. A. et al. A importância do fósforo no desenvolvimento inicial da planta.

Informações Agronômicas, n.95, 2001. 5p.

HIGA, R. C. V.; STURION, J. A. Capacidade de brotação em subgêneros e espécies de Eucalyptus. Série Técnica IPEF, v.11, n.30, p.23-30, 1997.

JESUS, R. M.; DIAS, G. B. N. Eucalyptus/ Leucaena mixture experiment - growth and yield. Série Técnica IPEF, v.39, p.41-46, 1988.

KIEHL, E. J. Manual de edafologia. São Paulo: Agronômica Ceres, 1979. 262p.

KUMAR, B. M. Intercropping teat with Leucena increases tree growft and modifies soil characteristics. Agroforest Systems, v.42, p.81-89, 1998.

MAFRA, A. L. et al. Produção de fitomassa e atributos químicos do solo sob cultivo em aléias e sob vegetação nativa de cerrado. Revista Brasileira de Ciência do Solo, v.22, n.1, p.43-48, 1998.

R. Árvore, Viçosa-MG, v.33, n.1, p.67-79, 2009
MAFRA, A. L. et al. Adição de nutrientes ao solo em sistema agroflorestal do tipo "cultivo em aléias" e em cerrado na região de Botucatu, SP. Scientia Forestalis, n.54, p.41-54, 1998.

MARTINS, S. G. et al. Avaliação de atributos físicos de um Latossolo Distroférrico sob diferentes povoamentos florestais. Cerne, v.8, n.1, p.32-41, 2002.

NÓBREGA, P. O. et al. Aporte de biomassa e nutrientes em sistema agroflorestal implantado em um Planossolo degradado no Estado do Rio de Janeiro. In: SIMPÓSIO NACIONAL DE RECUPERAÇÃO DE ÁREAS DEGRADADAS, 5. 2002, Belo Horizonte. Anais... Belo Horizonte: Sociedade Brasileira de Recuperação de Áreas Degradadas, 2002. p.518-520

NOVAIS, R. F.; BARROS, N. F.; NEVES, J. C. L. Interpretação de análise química do solo para o crescimento e desenvolvimento de Eucalyptus spp. Níveis críticos de implantação e de manutenção. Revista Árvore, v.10, n.1, p.105-111, 1986.

ODA, S. et al. Introdução de espécies/ procedências de Eucalyptus na região sub-úmida do Estado do Maranhão. Série Técnica IPEF, n.34, p.57-61, 1986.

PARROTA, J. A. Productivity, nutrient cycling, and succession in single- and mixed-species plantations of Casuarina equestifolia, Eucalyptus robusta and Leucaena leucocephala in Puerto Rico. Forest Ecology and Management, v.124, n.1, p.45-77, 1999.

PIOTTO, D. et al. Pure and mixed forest plantations with native species of the dry tropics of Costa Rica: a comparison of growth and productivity. Forest Ecology and Management, v.190, p.359-372, 2004.

RAMOS, I. S. et al. Dimensionamento da indústria cerâmica em Campos dos Goytacazes, RJ. In: CONGRESSOBRASILEIRODECERÂMICA, 47., 2003, João Pessoa. Anais... João Pessoa: 2003. CD ROM.

SANTIAGO, A. R. et al. Pure and mixed plantings of Eucalyptus in mining clay areas. In: EUCALYPTUS IN A CHANGING WORLD. PROCEDURE IUFRO CONFERENCE, 2004, Aveiro. Procedure... Aveiro: 2004. p.333-334p. 
SCHIAVO, J. A. et al. Comportamento de Acacia mangium Willd e Eucalyptus camaldulensis, em plantio puro e consorciado, em áreas degradadas pela extração de argila no Município de Campos dos Goytacazes. FERTIBIO 2004, Lages. Anais... Lages, 2004. CD-ROM.

TAIZ, L.; ZEIGER, E. Fisiologia Vegetal. 3.ed. Porto Alegre: Artmed, 2004.719p.

TOMÉ JÚNIOR, J. B. Manual para interpretação de análise do solo. Guaíba: Agropecuária, 1997. 246p.

TORRES NETTO, A. et al. Portable chlorophyll meter for the quantification of photosynthetic pigments, nitrogen and the possible use for assessment of the photochemical process in Carica papaya L. Brazilian Journal Plant Physiology, v.14, n.3, p.203-210, 2002.

TORRES, A. et al. Photosynthetic pigments, nitrogen, chlorophyll a fluorescence and SPAD502 readings in coffee leaves. Scientia Horticulturae, v.104, n.2, p.199-209, 2005.
VALICHESKI, R. R. et al. Atributos físicos do substrato remanescente da extração de argila no município de Campos dos Goytacazes, RJ. FERTIBIO 2003, Ribeirão Preto. Anais. Ribeirão Preto: 2003 CD-ROM.

VALICHESKI, R. R. Avaliação técnica e econômica de cenários de uso da terra em áreas degradadas por atividade de extração de argila em Campos dos Goytacazes, RJ. 2004. 138f. Dissertação (Mestrado em Produção Vegetal) - Universidade Estadual do Norte Fluminense, Campos dos Goytacazes, 2004. 138p.

VEZZANI, F. M.; TEDESCO, M. J.; BARROS, N. F. Alterações dos nutrientes no solo e nas plantas em consórcio de eucalipto e acácia negra.

Revista Brasileira de Ciência do Solo, v.25,p.225-231, 2001.

ZOTARELLI, L. et al. Calibração do medidor de clorofila Minolta SPAD-502 para uso na cultura do milho. Seropédica: Embrapa/CNPAB, 2002. (Boletim Técnico 55). 